\title{
Desarrollo de la teoría de la transición de Schlossberg y su aplicabilidad en el contexto de los estudiantes universitarios
}

\section{The development of Schlossberg's theory transition and its applicability in the context of university students}

\author{
Deps, Vera Lucia \\ Universidade Estadual do Norte Fluminense Darcy Ribeiro (UENF) \\ mielveh@gmail.com
}

Bernardo de Cara, Gláucio Roberto Universidade Estadual do Norte Fluminense Darcy Ribeiro (UENF)

(Brasil)

glauciorbc@gmail.com

Revista Cumbres Vol.5 №2

Versión impresa ISSN 1390-9541

Versión electrónica ISSN 1390-3365

http://investigacion.utmachala.edu.ec/revistas/index.php/Cumbres 


\title{
RESUMEN
}

Esta investigación tiene como objetivo presentar el desarrollo del modelo de análisis de la adaptación humana a la transición de Schlossberg y sus asociados, con énfasis en el enfoque de la caracterización del evento, así como el sistema de recursos potenciales (4S). Además, hay estudios que utilizaron este modelo para analizar el proceso de transición de estudiantes de pregrado. Por lo tanto, se realizó una revisión de la literatura de naturaleza cualitativa. La teoría se presenta en el aspecto psicosocial y postula que la transición se refiere a cuatro conjuntos de variables, relacionadas con la situación, las características individuales (auto), el apoyo y las estrategias. Se enfatiza la relevancia de los análisis bajo el aspecto psicosocial dada la reciprocidad de los efectos sobre el rendimiento del estudiante, sin embargo, existe la escasez de estudios en el contexto de transición en un contexto universitario.

Palabras clave: Transición, adaptación, estudiantes universitarios.

\begin{abstract}
This research aims to present the development of the model of analysis of human adaptation to the transition of Schlossberg and its associates, with emphasis on the approach to characterization of the event as well as the system of potential resources (4S's). In addition, we verify the studies that have been used of such a model for the analysis of the transition process of new students in undergraduate courses. For this purpose, a qualitative literature review was carried out. The theory presents itself in the psychosocial aspect and postulates that the transition refers to four sets of variables, related to the situation, the individual characteristics (self), the support and the strategies. The relevance of psycho-social analyzes is emphasized given the reciprocity of effects on the student's performance, however, it is noteworthy the scarcity of studies in the context of transition in a university context.
\end{abstract}

Keywords: Transition, adaptation, university students.

\section{Cumbres}




\section{INTRODUCCIÓN}

Desde la perspectiva psicosocial del desarrollo adulto, ingresar a un curso de nivel superior es una etapa importante en el curso de la vida de las personas. Se considera que, entre otras razones, esto se debe a los diversos cambios, ambos resultantes de la integración en una nueva experiencia educativa, cuando los estudiantes se enfrentan al requisito de adaptarse a las reglas, normas y expectativas (Almeida; Soares, 2004), así como los impactos de naturaleza evolutiva, en gran medida operados en la transición de la adolescencia a la edad adulta (Powers, 2010; Bejerano, 2014).

Los enfoques que analizan el proceso de transición en la educación superior (Tinto, 1999; Almeida; Soares; Ferreira, 2000; Ferreira, Almeida, Soares, 2001) presentan una fuerte relación entre los aspectos psicosociales y académicos. Por lo tanto, está claro que la forma en que el alumno pasa por el proceso de adaptación a la nueva realidad, en ambos aspectos, puede reflejarse positiva o negativamente en su desempeño.

Según lo presentado por Pinheiro (2004), aunque el problema de la adaptación a la universidad está incluido en las preocupaciones de los investigadores, son poco comunes las reflexiones completas a la luz de un marco teórico psicosocial dentro de esta transición. Almeida y Soares (2004) señalan que, además de los problemas relacionados con el aprendizaje y el rendimiento académico, es importante destacar otros tipos de variables más relacionadas con el yo de los estudiantes, ya que las tareas que enfrentan en una transición educativa no son están limitados exclusivamente a aquellos de naturaleza curricular.

En la década de 1980, Nancy K. Schlossberg desarrolló la teoría del análisis de la adaptación humana a la transición. Estaba constituido por una estructura sistemática que incorpora la noción de variabilidad para facilitar la comprensión de los eventos de transición. La teoría se presenta en el aspecto psicosocial y postula que la transición infiere cuatro conjuntos de variables, a saber: situación en la que ocurre el evento; características individuales (auto); apoyo recibido; y estrategias de afrontamiento.

A partir de esta suposición, este estudio utiliza una revisión de literatura de naturaleza cualitativa, a través de una síntesis de la teoría de la adaptación humana a la transición de Schlossberg y sus colaboradores, así como los estudios que la utilizaron en el análisis del proceso de transición de estudiantes en el contexto universitario.

Aunque algunos estudios (Coccarelli, 2010, Powers, 2010, Bejerano, 2014) han utilizado el modelo de análisis de Schlossberg y colaboradores y encontraron los impactos de la transición a la vida universitaria, estos hallazgos no se aplican completamente a las condiciones distintivas. de la realidad en la que se desarrolló la investigación. Según la literatura especializada (Anderson; Goodman; Schlossberg, 2012; Papalia; Feldman, 2013), los factores individuales, situacionales y contextuales afectan directamente el grado de impacto en el individuo en una circunstancia dada, por lo que es necesario ajustarlos de acuerdo con las especificidades de cada individuo. cada contexto 
Debido a la escasez de investigación sobre el tema, se cree que es posible proporcionar subsidios que puedan contribuir a futuras investigaciones con el objetivo de comprender mejor este proceso de transición, lo que puede contribuir al mejor uso de los recursos disponibles que pueden facilitar la adaptación y, en consecuencia, , promueve el mejor desempeño de los estudiantes.

\section{MATERIALES Y MÉTODOS}

\section{El camino del desarrollo de la teoría.}

La teoría de la transición humana de Nancy K. Schlossberg en 1981 se describió originalmente en un artículo titulado "Un modelo para analizar la adaptación humana a la transición", publicado en The Counseling Psychologist, Universidad de Maryland, EE. UU. En ese momento, los investigadores del curso de la vida estaban preocupados por la "importancia [...] de los eventos de transición que marcan el desarrollo humano, porque los perciben como determinantes de las transformaciones que ocurren con el tiempo" (Deps, 1994, p. 5).

A medida que el interés en el desarrollo adulto se ha multiplicado, se ha reconocido que: el crecimiento psíquico no termina con la adolescencia; A medida que las personas siguen su curso de vida, experimentan cambios y transiciones continuamente; y estos cambios a menudo resultan en nuevas redes de relaciones, nuevos comportamientos y nuevas autopercepciones. Desde este entendimiento, Schlossberg (1981) define la transición como un evento o no evento que resulta en un cambio significativo en las rutinas, roles sociales, relaciones y suposiciones sobre uno mismo y el mundo.

Desde su perspectiva, Schlossberg (1981) entiende el concepto de pasantía para adultos como un período de cambio y desarrollo multifactorial. Por lo tanto, las investigaciones no se limitan a un área específica de conocimiento, sino que constituyen el entrelazamiento de evidencia de diferentes líneas de observación.

Para elaborar su teoría, Schlossberg (1981) se basó en estudios que tratan sobre el desarrollo de adultos, como los de Levinson et al. (1978), Neugarten (1979), entre otros, de los cuales la autora refutó algunas concepciones y abstracciones de lo que parecía apropiado para su propósito. Posteriormente, el autor catalogó y categorizó una gran cantidad de variables que parecen afectar el resultado de la transición sobre el individuo. Las variables se compartimentaron en tres conjuntos de factores, a saber: "[...] las características de la transición en sí, las características de los entornos previos y posteriores a la transición y las características del individuo" (Deps, 1994, p. 14).

En 1984 Schlossberg lanzó su primer libro sobre el tema, Consejería para adultos en transición. Ante el surgimiento de nuevas perspectivas teóricas y transformaciones diversificadas en el panorama sociocultural, Anderson, Goodman y Schlossberg (2012) revisaron el trabajo hasta su cuarta edición, titulado "Orientando a los adultos en transición: vinculando la teoría de Schlossberg con la práctica "en un mundo diverso".

\section{Cumbres}


En resumen, el desarrollo de la teoría se justifica explícitamente por la comprensión de que cuando las personas pueden explorar completamente el tema de la transición, reconocer el significado subyacente y desarrollar un plan, es más probable que lidien más fácilmente con la etapa de transición. transición

Para trabajar en el proceso de transición, los autores estructuraron el modelo teórico en tres partes principales. La primera parte aborda el proceso de identificación de la naturaleza de la transición y también describe las fases de las Transiciones de Aproximación: identificación de transición y proceso de transición. La segunda parte presenta una estructura sistemática con los factores que los autores consideran que interfieren en el proceso de cambio (Hacer un balance de los recursos de afrontamiento: el sistema de las 4 S). La última parte ofrece contenido para el asesoramiento de transición para adultos (hacerse cargo: fortalecer los recursos).

El propósito de este estudio es enfatizar las partes que abordan la caracterización del evento y la identificación de la fase del proceso, así como el sistema de recursos potenciales (4S), para que podamos caracterizar y examinar, respectivamente, objeto de investigación.

El proceso y la caracterización de los eventos de transición en la teoría de Schlossberg et al.

Tal como se presenta, la aproximación de la transición está relacionada con la caracterización de la naturaleza de la transición y las fases que conforman el proceso.

La teoría predice que la caracterización de la transición involucra los conceptos de tipo, contexto e impacto. Estos se incorporaron a la estructura del esquema de observación, como se presenta en la Figura 1:

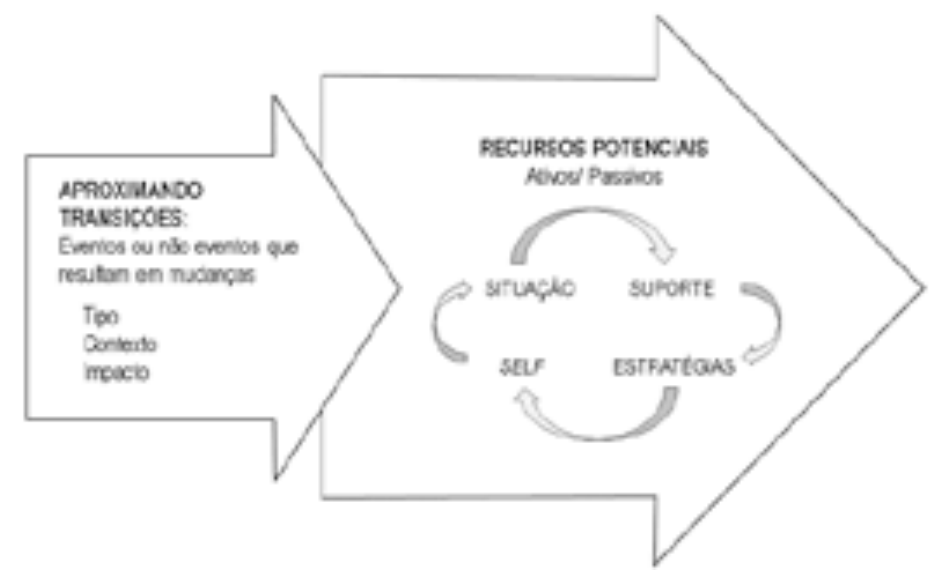

Fig.1 - El proceso de transición: cambio de reacciones a lo largo del tiempo Fuente: Anderson, Goodman y Schlossberg (2012) (adaptado).

Cuando se trata de escribir, está relacionado con la suposición de la ocurrencia o no del evento en la vida del individuo. Schlossberg, Waters y Goodman (1995) clasificaron y conceptualizaron las transiciones en tres tipos, a saber: i) transiciones tempranas; ii) no eventos; y iii) eventos imprevistos. Según la teoría, las transiciones anticipadas son aquellas que ocurren dentro de la 
expectativa esperada y se clasifican como eventos, por lo que un no evento se define como un evento que el sujeto esperaba que ocurriera, pero no sucede como se esperaba.

En el caso de las transiciones anticipadas, incluyen percepciones de ganancias y pérdidas que ocurren de manera predecible durante la vida de una persona, como resultado de eventos normativos, que generan cambios importantes en los roles. Las transiciones no anticipadas o no anticipadas son justo lo contrario de las transiciones anticipadas que consisten en eventos no planificados que no son predecibles.

El contexto se refiere a la relación de uno con la transición (personal, interpersonal o social) y el entorno en el que ocurre esta transición. Anderson, Goodman y Schlossberg (2012) señalan que aunque los factores contextuales pueden tener influencias directas e indirectas en una transición, estos factores también pueden influir en la percepción individual de las opciones disponibles para uno mismo. La transición puede involucrar la autoestima, las amistades, la familia, el trabajo, la salud y el estado socioeconómico del sujeto; así como aspectos relacionados con el contexto sociocultural, político y económico.

El impacto se refiere a la evaluación del grado en que una transición que altera la vida (Anderson; Goodman; Schlossberg, 2012). En este sentido, se enfatiza la subjetividad del individuo y el contexto. Pinheiro (2004) demuestra que ninguna transición es intrínsecamente predecible o impredecible, es decir, lo que puede ser un cambio inesperado para una persona puede ser predecible para otra. Además, lo que puede constituir una transición por la ausencia de un evento para uno puede ser una decisión planificada para otro. Cruz (2008) señala que la transición puede ser evaluada por el individuo tanto positiva como negativamente, es decir, el mismo evento no siempre se evalúa de la misma manera. Según el autor, el impacto también depende de la forma en que se produce la transición (ya sea progresiva o inmediata) y su sincronización (si se produce más de una transición simultáneamente).

Con respecto al proceso de transición, Schlossberg, Waters y Goodman (1995) lo entienden como una composición de tres etapas, que se conceptualiza como "entrar", "pasar" y "salir". Pinheiro (2004) los traduce, respectivamente, como: la entrada en la transición, la permanencia en la transición y la salida o finalización.

Como lo menciona Vieira (2012), el modelo integrador propuesto por los autores considera que, independientemente de la transición, el punto de partida puede ser una iniciación o incluso un final. La iniciación ocurre cuando los individuos se enfrentan a una nueva experiencia en la que se requiere adaptación a las reglas, normas y expectativas, mientras que la transición resultante de un final se caracteriza por el abandono de un contexto, personas o formas familiares. interacción que generalmente promueve cierta desorganización.

Después del inicio, sigue el período de reevaluación, que se relaciona con el equilibrio y el deterioro. Una vez en una nueva situación, las personas deben aprender a equilibrar sus actividades con otras áreas de sus vidas a me-

\section{Cumbres}


dida que avanzan en la transición. Para Anderson, Goodman y Schlossberg (2012), este tiempo intermedio puede evocar en el tema nuevas preguntas sobre la transición.

La salida, o el final, puede verse como el final de la transición. Esta etapa se refiere al momento en que la transición se integra y se restablece un período de estabilidad. Eventualmente, puedes pensar en lo que viene después.

De hecho, durante el proceso de transición, los roles, las relaciones, los supuestos y las rutinas anteriores cambian y se desarrollan gradualmente nuevas formas de ser y actuar hasta el punto en que el individuo se adapta a la nueva fase y integra la transición en su camino existencial (Vieira, 2012). Por lo tanto, la transición es un proceso extenso en el tiempo, que incluye fases de asimilación y evaluación continua, al mismo tiempo que el individuo comienza, pasa y abandona este proceso (Anderson; Goodman; Schlossberg, 2012).

\section{Recursos potenciales (El sistema de las 4 S)}

A pesar de la variabilidad de la naturaleza de las transiciones y las características individuales, Schlossberg, Waters y Goodman (1995) consideran que la estructura para comprender a los individuos en transición es estable $\mathrm{y}$, a partir de esto, es posible definir un patrón relativamente consistente que permita una mayor comprensión del proceso, que puede conducir a la reflexión de estrategias de afrontamiento apropiadas.

Como se mencionó, la nueva estructura sintetiza los factores del modelo de análisis de adaptación humana para la transición a cuatro categorías de variables: situación, características individuales, apoyo y estrategias. Dichos términos en inglés comienzan con la letra "s": situación, autocontrol, apoyo y estrategias, respectivamente. Para Anderson, Goodman y Schlossberg (2012), estos factores se toman como recursos potenciales y constituyen activos (recursos) y pasivos (déficits), que interactúan en una relación dinámica e inestable durante el proceso de transición. Estas variables se reorganizaron en un esquema, como se muestra en la figura 2.

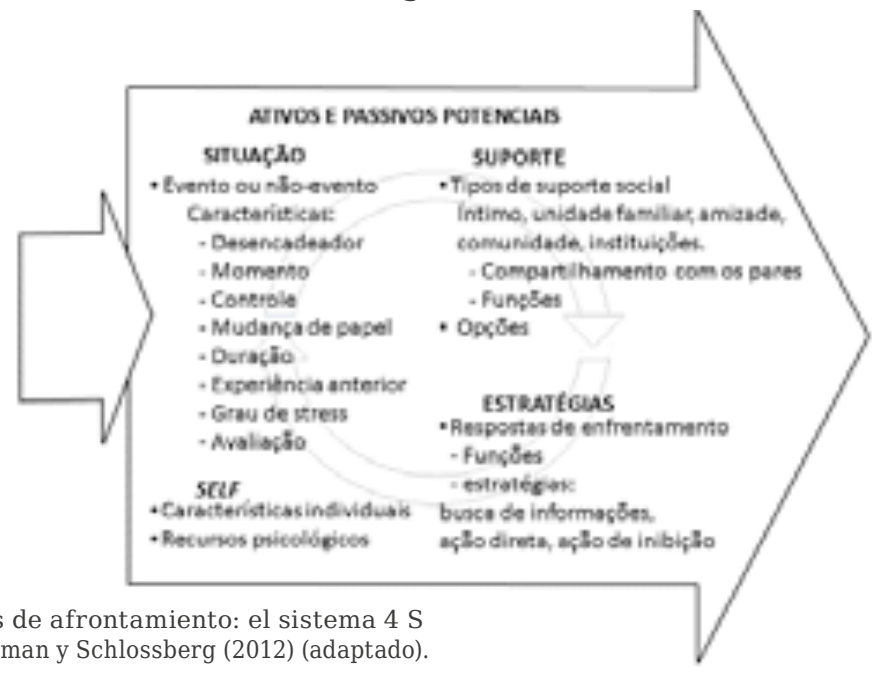

Fuente: Anderson, Goodman y Schlossberg (2012) (adaptado). 
Según la teoría, las variables del factor de situación describen las propiedades de la transición y comprenden: el desencadenante de la transición; el momento (predicho o imprevisto, el momento bueno o malo); control sobre aspectos situacionales; el papel cambiante que viene con la transición; la duración (permanente, temporal o incierta); la experiencia de situaciones anteriores similares a la transición actual y sus respuestas producidas; el estres y la evaluación individual de la transición.

El segundo factor, el yo, viene en dos variables principales: características individuales y recursos psicológicos. Las características individuales incluyen el estado socioeconómico, el género y la orientación sexual, la edad y la etapa de la vida, y la etnia / cultura, mientras que los recursos psicológicos infieren las características de la personalidad que intervienen en el proceso de transición. tales como el desarrollo del ego, el optimismo, la autoeficacia, el compromiso y la entrega de valores, así como la espiritualidad y la capacidad de recuperación.

El tercer factor, el apoyo, se refiere a las personas y otros activos que ayudan al individuo durante la transición. Esta categoría representa componentes sociales y de comunicación, tales como: relaciones íntimas, unidades familiares, red de amigos, instituciones y comunidades, así como sus roles asignados; como recursos disponibles (opciones).

Con respecto al cuarto y nuevo factor incluido, las estrategias, se relaciona con las acciones o tácticas que un individuo usa para lidiar con una transición. En este factor, se consideran las creencias positivas y la resolución de problemas a través de las habilidades sociales, y se puede utilizar el apoyo social o el uso de los recursos materiales disponibles. Según la teoría, este factor está directamente relacionado con los recursos psicológicos de la autoestima y el dominio de las personas.

Para observar el proceso de transición a la teoría, por lo tanto, propone la aplicabilidad de un análisis simultáneo de los aspectos individuales, sociales y ambientales, así como la compleja relación existente entre estos aspectos, basada en suposiciones desde la perspectiva del desarrollo adulto y en otros. modelos que enfatizan el enfoque de transición en el curso de la vida.

\section{RESULTADOS}

\section{La aplicabilidad del modelo en la transición a la entrada a la educación superior}

Esta parte consiste en una breve descripción de tres estudios realizados en los últimos diez años, utilizando el modelo de análisis de transición humana de Schlossberg, Waters y Goodman (1995) en el contexto de estudiantes universitarios en diferentes situaciones.

Coccarelli (2010) analizó el proceso de transición de la escuela secundaria a la universidad para los estudiantes que se identificaron con discapacidades de aprendizaje. Los datos fueron recolectados en dos universidades

\section{Cumbres}


públicas en el suroeste de los Estados Unidos. Se entrevistó a un total de cinco estudiantes y un miembro del personal del departamento de servicio de discapacidad para estudiantes. Su estudio destaca que las características individuales tuvieron un mayor impacto en el proceso de transición de estos estudiantes. El autor no presenta conclusiones sobre el proceso de transición en sí, sin embargo, menciona las recomendaciones sugeridas por los entrevistados, que podrían contribuir a este proceso. Entre estas recomendaciones, el autor enfatiza alentar a los estudiantes a establecer sus metas a corto y largo plazo, así como el apoyo académico para guiarlos, especialmente durante el proceso de transición.

Powers (2010) exploró el proceso de transición de 14 estudiantes que abandonaron la universidad después de dos años de estudio. Desde una perspectiva personal e institucional, su estudio encontró que a medida que los estudiantes progresaban en la transición, aumentaba la percepción de responsabilidad. La autora señala que la mayoría de los participantes en su investigación iniciaron la transición con un número razonable de condiciones propicias, sin embargo, durante el proceso, surgieron dificultades que superaron la capacidad de las personas para superarlas, lo que resultó en evasión.

Bejerano (2014) investigó el proceso de transición a la universidad de 197 estudiantes de secundaria recientes. Observó cómo el apoyo de los compañeros, la familia y los maestros, así como las características personales, el compromiso y las estrategias se relacionan con la adaptación del estudiante. Los resultados revelaron que la autoestima era el predictor más fuerte de ajuste, seguido del compromiso, el apoyo del maestro, los compañeros y la familia. Contrariamente a su hipótesis, las estrategias no fueron predictores significativos en este proceso.

\section{CONCLUSIONES}

Como se enfatizó a lo largo de este artículo, el modelo para el análisis de la transición humana a la adaptación propuesto por Schlossberg y sus asociados describe un conjunto de variables que ella considera que interfieren con este proceso siempre que resulten en cambios en las rutinas, roles sociales, relaciones y relaciones. en los supuestos sobre ti y el mundo.

Aunque este estudio ha destacado la descripción de su modelo y su aplicabilidad en el contexto de la transición de los estudiantes a la universidad, debe enfatizarse que su teoría puede aplicarse a varios tipos de transición, como la jubilación (Deps, 1994), ingresar al mercado laboral (Vieira, 2012), entre otros, tanto en eventos normativos como no normativos, siempre que cumpla los criterios relacionados con las características de las transiciones. Como se describe en su alcance de revisiones, particularmente en aquellas partes que ofrecen contenido para el asesoramiento de adultos en transición (Encargado: fortalecer los recursos), el marco teórico se puede utilizar para el análisis individual y colectivo. 
La teoría se destaca por su perspectiva multifactorial en el análisis de los procesos de transición. Es de destacar que su elaboración se deriva de la integración de una serie de otras teorías que impregnan la coyuntura de su desarrollo. Por lo tanto, su enfoque no se limita a un área particular de conocimiento, sin embargo, se entrelaza en diferentes líneas de observación, lo que contribuye a una mejor comprensión de los objetos de investigación a los que se aplican.

Con respecto al contexto de la transición de los estudiantes a la universidad, desde una revisión sistemática de la literatura, hay una escasez de estudios desde perspectivas tan amplias como la que ofrece la teoría de Schlossberg. Sin embargo, lo que encontró la investigación de Coccarelli (2010), Powers (2010) y Bejerano (2014) validan el alcance teórico en el contexto sociohistórico actual y demuestran la importancia de los estudios que pueden comprender mejor la realidad de los estudiantes que estudian. alcanzar este nivel de educación. Con esto será posible contribuir a la calidad en el proceso de transición, logrando así un mejor desempeño en los alunos.

\section{REFERENCIAS BIBLIOGRÁFICAS}

Almeida, L., Soares, A. P. (2004). Os estudantes universitários: sucesso escolar e desenvolvimento psicossocial. In: Elizabeth, M., Soely, P. (Eds.). Estudante universitário: características e experiências de formação. (pp. 1540). Taubaté: Cabral Editora e Livraria Universitária.

Almeida, L., Soares, A., Ferreira, A. G. (2000). Transição e adaptação à universidade: Apresentação de um Questionário de Vivências Acadêmicas (QVA). Psicologia, 14, 189-2008. Disponível em: <http://www.scielo.mec.pt/ scielo.php?script=sci_arttext\&pid=S0874-20492000000200005 >. Acesso em: 10 jun. 2018.

Anderson, M. L., Goodman, J., Schlossberg, N. K. (2012). Counseling adults in transition: linking Schlossberg's theory with practice in a diverse world. New York: Springer Publishing Company.

Bejerano, A. (2014). An examination of the role of social support, coping strategies, and individual characteristics in students' adaptation to college. $188 \mathrm{f}$. Tese (Communication Studies) - Department of Educational Administration, University of Nebraska at Lincoln, Nebraska. Disponível em: <https://digitalcommons.unl.edu/cgi/viewcontent.cgi?article=1028\&context=commstuddiss >. Acesso em: 10 jun. 2018.

Cruz, M. A. C. (2008). Ansiedade e bem-estar na transição para o ensino superior: o papel do suporte social. Dissertação (Mestrado em Psicologia) - Faculdade de Psicologia e de Ciências da Educação, Universidade do Porto, Porto, Portugal. Disponível em: <https://repositorio-aberto.up.pt/handle/10216/23383>. Acesso em: 05 ago. 2018.

Deps, V. L. (1994). A transição à aposentadoria na percepção de professores recém-aposentados da Universidade Federal do Espírito Santo. Tese (Doutorado em Educação) - Faculdade de Educação, Universidade Estadual de Campinas, Campinas, SP, Brasil.

\section{Cumbres}


Ferreira, J. A., Almeida, L. S., Soares, A. P. C. (2001). Adaptação académica em estudante do 1o ano: diferenças de gênero, situação de estudante e curso. Psico-USF, 6, 1-10. Disponível em: <http://www.scielo.br/scielo. php?pid=S1413-82712001000100002\&script=sci_abstract\&tlng $=$ pt $>$. Acesso em: 05 jul. 2018.

Levinson, D.J., Darrow, C.N., Klein, E.D., Levinson, M.G., McKee, B. (1978). The seasons of man's life. Nova Iork: Knopt.

Neugarten, B.L. (1979). Time, age, and the life cycle. [s.l]: Américan Journal of psychiatry.

Papalia, D. E., Feldman, R. D. (2013). Desenvolvimento humano. Porto Alegre: AMGH.

Pinheiro, M. R. M. (2004). O princípio depois de um fim. Aprender, 29, 9-20. Disponível em: <http://legado.esep.pt/aprender/index.php/component/phocadownload/category/31-revista-aprender-n-29?download=604:aprender-29-a2>. Acesso em: 05 jul. 2018.

Powers, M. (2010). Applying Schlossberg's transition theory to nontraditional male drop-outs. Tese (Educational Studies/ Educational Leadership in Higher Education) - Department of Educational Administration, University of Nebraska at Lincoln, Nebraska. Disponível em: < https://digitalcommons. unl.edu/cehsedaddiss/19/?utm_source=digitalcommons.unl.edu\%2Fcehsedaddiss\%2F19\&utm_medium=PDF\&utm_campaign=PDFCoverPages>. Acesso em: 10 jun. 2018.

Schlossberg, N. K. (1981). A model for analyzing human adaptation to transition.Counseling Psychologist, 9, 2-18. Disponível em: <http://journals.sagepub.com/doi/abs/10.1177/001100008100900202>. Acesso em: 12 mar. 2018.

Schlossberg, N. K., Waters, E. B., Goodman, J. (1995). Counseling adults in transition: linking practice with theory. Nova Iork: Springer Publishing Company.

Tinto, V. (1999). Taking retention seriously: rethinking the first year of college. Nacada Journal, 19, 5-9. Disponível em: <http://www.nacadajournal.org/ doi/pdf/10.12930/0271-9517-19.2.5>. Acesso em: 10 jun. 2018.

Vieira, D. A. (2012). Transição do ensino superior para o trabalho: o poder da auto-eficácia e dos objetivos profissionais. Porto: Edições politema. 